\title{
Immunosuppressive activity in patients with active chronic hepatitis and primary biliary cirrhosis treated with azathioprine
}

\author{
GREGORY WHELAN ${ }^{1}$ AND SHEILA SHERLOCK \\ From the Department of Medicine, Royal Free Hospital, London
}

SUMMARY The rosette inhibition test was used to measure serum immunosuppressive activity after azathioprine $(1.5 \mathrm{mg} / \mathrm{kg})$ in 20 patients with liver disease (10 with active chronic hepatitis, six with primary biliary cirrhosis, and four with miscellaneous hepatic disorders) and in nine healthy normal volunteers. Where possible testing was repeated after one week and after at least two months of treatment. The titre of immunosuppression was related to the degree of impairment of hepatic function, and was found to be low in those with severe and normal in those with mild disturbance of hepatic function. Titres were unrelated to the presence of immunological abnormalities such as smooth muscle antibodies. In patients treated for at least two months there was no relationship between the initial titre of immunosuppressive activity and the response to treatment. Thus some patients improved despite failure to develop immunosuppressive activity, while others deteriorated even though immunosuppressive activity was normal when first studied. The development of immunosuppressive activity after azathioprine seems to be dependent on adequate hepatic function and the favourable response of some patients with liver disease to the drug may not be due entirely to immunosuppression.

The antimetabolites, 6-mercaptopurine and azathioprine, are used in the therapy of patients with liver disease, especially in those with active chronic hepatitis. Encouraging results have been obtained in some cases (Mackay, Weiden, and Ungar, 1964; Mackay, 1968; Mistilis and Blackburn, 1967; Page, Good, and Pollara, 1969), especially in young patients with hypergammaglobulinaemia (Page et al, 1969). Since azathioprine has to be metabolized in order to be fully active (Elion, 1967), the observation that a favourable response to therapy was obtained with quite small doses of the drug (Mistilis and Blackburn, 1967) aroused considerable interest and suggested that the metabolism of these drugs is different in patients with liver disease. Normally 6-mercaptopurine and azathioprine are converted soon after administration to thioinosinic acid and

\footnotetext{
${ }^{1}$ Dr Whelan was supported by a Wellcome Trust research fellowship. His present address is: Department of Medicine, University of Melbourne, St Vincent's Hospital, Fitzroy, Victoria, Australia
}

Please address requests for reprints to: Professor S. Sherlock, Department of Medicine, Royal Free Hospital, Gray's Inn Road, London, WC1X 8LF.

Received for publication 29 August 1972. its derivatives (Elion, 1967, 1968). This minor pathway of metabolism is important since these particular metabolites are considered to be primarily responsible for the action of these antimetabolite drugs (Elion, 1967a, 1967b, 1969). Most of the drug is, however, metabolized via a catabolic pathway resulting in the formation of a variety of inactive end-products which appear in the urine within hours of administration (Elion, 1967a and b). Concentrations of total drug in the blood rarely exceed 1 $\mu \mathrm{g} / \mathrm{ml}$ and at that level currently employed methods have not been sufficiently sensitive to determine accurately the distribution of metabolized and unchanged drug (Elion, 1967b). Recently this problem has been approached by using the rosette inhibition test to assess the level of circulating biologically active drug after the administration of antimetabolite drugs such as azathioprine (Bach, Dardenne, and Fournier, 1969a).

The mechanism of spontaneous rosette formation is uncertain. It is postulated by Bach that antigenic sites on the red cell membranes adhere to antibodies on the cell membranes of the lymphocytes involved in antibody production (Bach et al, 1969; 
Bach and Dormont, 1971). These authors also suggest that the rosette-forming cell is thymusdependent and antigen-sensitive (Bach and Dormont, 1971). Antilymphocyte globulin is felt to coat lymphocytes and thus prevent adherence of red cells. Whether the immunosuppressive drugs act similarly is uncertain. However the action is not due to direct cytotoxicity as the effect is reversible at $37^{\circ} \mathrm{C}$ upon washing the lymphocytes involved (Bach, 1970). Although the mechanism involved in the rosette inhibition test may be obscure, it is regarded as a good index of immunosuppressive potency. This has been best shown with antilymphocyte globulin (ALG) where survival graphs and rosette inhibition titres in animals show a good correlation (Bach, Dormont, Dandenne, and Balner, 1969).

Since the liver contains enzyme systems involved in the anabolic (eg, hypoxanthine-guanine phosphoribosyl transferase) as well as the degenerative pathway, eg, xanthine oxidase (Elion, 1967), it seemed reasonable to postulate that the presence of significant liver disease could result in alterations to the metabolism of azathioprine. Therefore, the metabolism of azathioprine has been studied in patients with chronic liver disease, utilizing the rosette inhibition test to measure the serum immunosuppressive activity of the drug.

\section{Patient Population}

The 16 patients with active chronic hepatitis (10 patients) or primary biliary cirrhosis (six patients) were commencing therapy with azathioprine. One patient (no. 16) was taking diuretics and two patients (nos. 6 and 16) had taken, within the previous three weeks, drugs known to be associated with the enhancement of drug-metabolizing enzymes. Renal function, as assessed by urine analysis, estimation of blood urea, serum creatinine, and creatinine clearance was always normal, although this subsequently deteriorated in two patients (nos. 9 and 16).

PATIENTS WITH ACTIVE CHRONIC HEPATITIS The ages of the 10 patients with active chronic hepatitis ranged from 12 to 50 years. Symptoms had commenced nine months to 11 years previously. Liver biopsy in all showed chronic aggressive hepatitis with or without cirrhosis. All but three patients (nos. 2, 7, and 10) had previously received ACTH or corticosteroids but none was presently taking these drugs. An attempt has been made to assess objectively overall hepatic function in each patient. Since no good absolute criteria are available, the three clinicians who were responsible for the follow-up care of these patients were asked to grade overall hepatic function on the basis of clinical features and biochemical findings without knowledge of the results of the rosette inhibition test. The criteria adopted to define good, fair, and bad hepatic function are shown in Table II. Liver biopsies were not used in the assessment because of the variable size of specimens and the risk of sampling error (Scheuer, 1968). Gamma globulin levels were elevated in all but one patient (no. 6). Smooth muscle antibody was positive in seven of 10 patients (nos. 1, 2, 4, 5, 8, 9, 10), antinuclear factor positive in five (nos. 2, 5, 8, 9, 10), and antimitochondrial antibody and the LE cell phenomenon positive in one each (no. 3 and no. 5 respectively). These criteria of disturbed immunity were not used in the assessment of hepatic function since they do not necessarily reflect the activity of the liver disease. Australia antigen was not detected in the serum of any patient.

\section{PATIENTS WITH PRIMARY BILIARY}

CIRRHOSIS

The six patients with primary biliary cirrhosis were diagnosed by the history, clinical features, and the results of investigations shown in Table III. Their ages ranged from 35 to 65 years and apart from one patient who was asymptomatic (no. 12) they had had symptoms for one to eight years. None were taking cholestyramine at the time of the study. These patients cannot readily be divided into several groups with regard to their hepatic function. However, since deep jaundice, ascites, and hypoalbuminaemia are considered poor prognostic signs, it can be seen that three patients (nos. 14, 15, and 16) had advanced liver disease, whereas hepatic function was better preserved in the other three patients (nos. 11, 12, and 13).

\section{PATIENTS WITH MISCELLANEOUS LIVER}

DISEASE

Details of these four patients, including their diagnoses, results of their biochemical tests, and liver biopsies, are given in Table IV. This group was studied to determine if liver disease without positive markers of immunological disturbance behaved in a similar fashion. Two patients (nos. 19 and 20) had ascites and hypoalbuminaemia but were without the marked elevations of serum aspartate aminotransferase (Ast) usually found in the group with active chronic hepatitis. A further patient (no. 17) had mild hyperbilirubinaemia but otherwise normal liver function tests and a normal liver biopsy. The fourth patient (no. 18) was studied twice. Initially this was done early in her illness when she had marked abnormalities of hepatic function. On the second occasion, several weeks later, her hepatic function had markedly improved. 


\section{Procedure}

Peripheral venous blood samples were taken on at least one occasion before commencing therapy and then at frequent intervals over the first 24 hours following the initial oral single dose of azathioprine $(1.5 \mathrm{mg} / \mathrm{kg}$ body weight). Some patients were restudied one week after beginning therapy and again after at least two months of continuous daily medication. Observations were also made in a group of nine normal volunteer subjects consisting of male and female students, medical and laboratory personnel, ranging in age from 17 to 50 years, after a single oral dose of azathioprine $(1.5 \mathrm{mg} / \mathrm{kg})$.

Selected patients and some corresponding age- and sex-matched controls were restudied after a single oral dose of azathioprine $3.0 \mathrm{mg} / \mathrm{kg}$ body weight.

\section{PREPARATION OF SAMPLES}

Naturally occurring anti-sheep haemagglutinating antibodies were removed from the serum, initially by absorbing each serum sample a total of five times with half its volume of sheep red cells, previously washed thrice in saline, at $37^{\circ} \mathrm{C}$ for 30 minutes. Recently it was found that removal of these antibodies could be achieved with a similar degree of efficiency in an ultrafiltration apparatus using an XM-50 Amicon dialysing membrane (Bach, J. F., personal communication), and this procedure was adopted. All serum samples were coded and stored at $-20^{\circ} \mathrm{C}$ until tested.

\section{MEASUREMENT OF IMMUNOSUPPRESSIVE}

ACTIVITY OF AZATHIOPRINE IN SERUM

The immunosuppressive activity of each serum sample was determined utilizing the rosette inhibition test of Bach and Antoine (Bach et al, 1969a). Spontaneous rosette formation will occur when mouse spleen cells are incubated with sheep red cells and centrifuged together for five minutes at 150 to $200 \mathrm{~g}$. Serum containing active metabolites will inhibit rosette formation if incubated with mouse spleen cells before adding the sheep red cells (Bach et al, 1969a).

All serum samples were tested under code and therefore without knowledge of the patient's clinical state and liver function tests. C 57 black mice were used as a source of spleen cells. Disposable plastic tubes and pipettes were used to minimize the problems from inadequately cleaned glassware. In performing the test serial dilutions of serum in Hanks BSS (calcium-free) from $1 / 2$ to $1 / 256$ were made and coded to minimize subjective observer error during subsequent counting. Of all dilutions, $0.1 \mathrm{ml}$ was then incubated with $0.6 \mathrm{ml}$ of the spleen cells preparation containing $6 \times 10^{6}$ spleen cells in
Hanks BSS for 90 minutes at $37^{\circ} \mathrm{C}$ in a water bath. Two additional tubes containing only Hanks BSS without serum served as controls. Rosette formation was then achieved by the addition of $0.25 \mathrm{ml}$ of $1 \%$ sheep red cells in saline and centrifugation of each tube for five min at $150 \mathrm{~g}$ at $4^{\circ} \mathrm{C}$. Cells were then re-suspended by gentle to and fro rotation of the tubes. Since any delay in counting at this stage results in a decline in the number of rosettes observed, $0.4 \mathrm{ml}$ of $4 \%$ formaldehyde in saline was then added to each tube after the resuspension process was completed (preliminary testing had previously shown that this step stabilized the rosette count for at least six hours). Cell counting was performed using a haemocytometer and the number of rosettes present per 1000 spleen cells in each tube was noted. The code was then broken and the results obtained were expressed as a percentage of control values. The titre of immunosuppressive activity of each sample was expressed as that dilution of serum which inhibited more than $25 \%$ of the rosette-forming cells. Preliminary studies in two controls showed that the peak of activity in serum was at one hour after the administration of azathioprine. In two patients no rise of immunosuppressive activity was observed after one hour and thus routinely only samples taken before treatment at one hour and at 24 hours after azathioprine were examined.

STATISTICAL ANALYSIS

The Mann Whitney test for nonparametric analytical procedures (Siegel, 1956) was used for evaluation of these experiments.

\section{Results}

1 SUBJECTS GIVEN AZATHIOPRINE $1.5 \mathrm{Mg} / \mathrm{KG}$ BODY WEIGHT

Normal subjects

In the nine subjects with normal hepatic function the

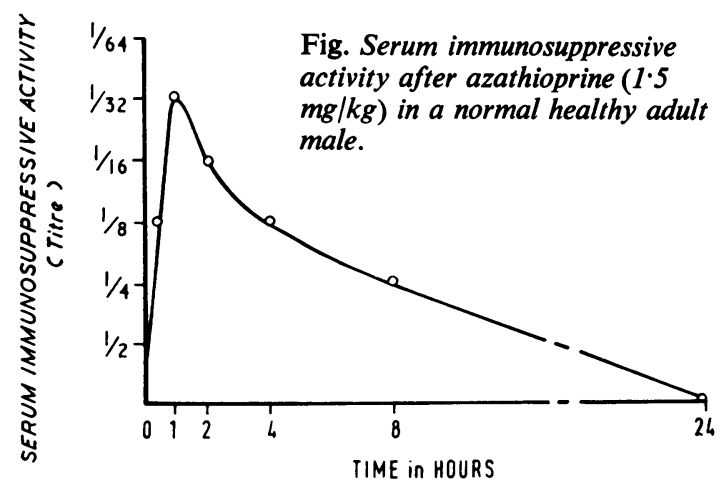


titre of immunosuppressive activity after one hour of administration of azathioprine was achieved at a dilution of $1 / 16$ or $1 / 32$. Pretreatment titres varied from undetectable to $1 / 4$ (Table I). Immunosuppressive activity declined with time and in every case returned to pretreatment values within 24 hours (see Fig.).

\begin{tabular}{llccc}
\hline Subject & $\begin{array}{l}\text { Azathioprine } \\
(\mathrm{mg} / \mathrm{kg})\end{array}$ & \multicolumn{3}{c}{ Titre of Immunosuppressive Activity } \\
\cline { 3 - 5 } \cline { 3 - 4 } & & Before Dose & One Hour & 24 Hours \\
\hline A & 1.5 & $1 / 2$ & $1 / 16$ & $1 / 2$ \\
& 3.0 & $1 / 2$ & $1 / 64$ & $1 / 2$ \\
B & 1.5 & $<1 / 2$ & $1 / 16$ & $<1 / 2$ \\
C & 1.5 & $<1 / 2$ & $1 / 32$ & $<1 / 2$ \\
D & 1.5 & $<1 / 2$ & $1 / 16$ & No sample \\
E & 1.5 & $1 / 4$ & $1 / 32$ & $1 / 4$ \\
& 3.0 & $1 / 4$ & $1 / 128$ & $1 / 4$ \\
F & 1.5 & $1 / 2$ & $1 / 16$ & $1 / 2$ \\
& 3.0 & $1 / 2$ & $1 / 64$ & $1 / 2$ \\
G & 1.5 & $<1 / 2$ & $1 / 32$ & $<1 / 2$ \\
H & 1.5 & $1 / 4$ & $1 / 32$ & $1 / 4$ \\
& 3.0 & $1 / 4$ & $1 / 64$ & $1 / 4$ \\
I & 1.5 & $1 / 2$ & $1 / 16$ & $1 / 2$ \\
\hline
\end{tabular}

Table I Immunosuppressive activity in normal subjects after azathioprine

Patients with active chronic hepatitis (Table II)

Hepatic function was graded as good in three, fair in four, and bad in three patients. This group attained a mean level of immunosuppressive activity in the serum of $1 / 8$, which was significantly less than that reached by the control group $(P=0.05)$. However, the three patients with good hepatic function were able to attain normal levels of serum immunosuppressive activity within one hour of administration of azathioprine reaching a titre of $1 / 16(P>0 \cdot 1)$. Of the seven patients with fair or bad hepatic function, six failed to elevate their titres and although a rise in titre was observed in the seventh patient, it did not reach the values obtained in normal. Thus, in patients with active chronic hepatitis with fair or bad hepatic function, the level of immunosuppressive activity was significantly lower than that obtained in normal controls $(P<0.001)$. On retesting again after one week of therapy these values in each patient were substantially unaltered. No major alteration in hepatic function had been noted.
Patients with primary biliary cirrhosis (Table III) Three patients had advanced liver disease (nos. 14, 15 , and 16). All were clinically jaundiced and two of them had marked hypoalbuminaemia. These three patients were unable to raise their titres above pretreatment levels. When initially tested two of the three patients with less severe liver disease from whom specimens were available for testing were able to achieve normal titres of immunosuppressive activity (nos. 11, 13). One week later there was no alteration in response to azathioprine among those patients retested.

\section{Patients with miscellaneous liver diseases}

Results of the rosette inhibition tests performed on these patients are shown in Table IV. The two patients with well advanced liver disease (nos. 19 and 20) failed to develop normal immunosuppressive activity. Patient no. 18, while in an acute phase of viral hepatitis, also failed to develop normal titres. However, during her convalescence as hepatic function improved, her titre came into the normal range. Patient no. 17 with normal liver functions, apart from a mildly elevated serum bilirubin, was able to develop a normal titre of immunosuppressive activity.

Thus, on initial testing, seven studies were performed in patients with only mild impairment of liver function and these patients were able to develop normal levels of serum immunosuppressive activity $(\mathrm{P}>0 \cdot 1)$. However, the 13 patients with fair or bad hepatic function were seldom able to raise their titre of serum immunosuppressive activity and levels reached were always less than normal $(\mathrm{P}<0.001)$.

\section{STUDIES AFTER PROLONGED}

\section{ADMINISTRATION OF AZATHIOPRINE}

Patients with active chronic hepatitis (Table II)

The study was repeated in six patients after at least two months of therapy. The other four patients (nos. 3, 6, 7, and 8) were no longer available. Hepatic function had deteriorated in three patients (nos. 1, 2, and 9). One patient (no. 1) had stopped taking the drug before his deterioration. Of the three patients who had improved two now had good

\begin{tabular}{|c|c|c|c|c|c|c|c|}
\hline $\begin{array}{l}\text { Hepatic } \\
\text { Function } \\
\text { (Grade) }\end{array}$ & Point Score & Clinical State & $A s T(i u / l)$ & $\begin{array}{l}\text { Albumin } \\
(\mathrm{g} / 100 \mathrm{ml})\end{array}$ & $\begin{array}{l}\text { Bilirubin } \\
(\mathrm{mg} / 100 \mathrm{ml})\end{array}$ & $\begin{array}{l}\text { Thrombotest } \\
(\%)\end{array}$ & $\begin{array}{l}\text { Overall Point } \\
\text { Score }\end{array}$ \\
\hline
\end{tabular}

The criteria used for grading hepatic function

${ }^{1}$ The physical activity assessed was the ability to do housework, attend school, or earn a living. 


\begin{tabular}{|c|c|c|c|c|c|c|c|c|c|c|c|c|c|c|c|}
\hline \multirow[t]{2}{*}{ No. } & \multirow[t]{2}{*}{ Sex } & \multirow[t]{2}{*}{$\begin{array}{l}\text { Age } \\
(y r)\end{array}$} & \multirow[t]{2}{*}{$\begin{array}{l}\text { Time of } \\
\text { Study }\end{array}$} & \multirow[t]{2}{*}{$\begin{array}{l}\text { Azathio- } \\
\text { prine } \\
(\mathrm{mg} / \mathrm{kg})\end{array}$} & \multicolumn{3}{|c|}{$\begin{array}{l}\text { Titre of Immunosuppressive } \\
\text { Activity }\end{array}$} & \multirow[t]{2}{*}{$\begin{array}{l}\text { AsT } \\
(i u / l)\end{array}$} & \multirow[t]{2}{*}{$\begin{array}{l}\text { Bilirubin } \\
(m g / 100 \\
m l)\end{array}$} & \multirow[t]{2}{*}{$\begin{array}{l}\text { Albumin } \\
(\mathrm{g} / 100 \\
\mathrm{ml})\end{array}$} & \multirow[t]{2}{*}{$\begin{array}{l}\text { Throm- } \\
\text { botest } \\
(\%)\end{array}$} & \multirow[t]{2}{*}{$\begin{array}{l}\gamma-G l o b u- \\
\operatorname{lin}(\mathrm{g} / \\
100 \mathrm{ml})\end{array}$} & \multirow{2}{*}{$\begin{array}{l}\text { Alkaline } \\
\text { Phos- } \\
\text { phatase } \\
\text { (KA } \\
\text { units) }\end{array}$} & \multirow{2}{*}{$\begin{array}{l}\text { Hepatic } \\
\text { Function } \\
\text { (grade) }\end{array}$} & \multirow[t]{2}{*}{ Comment } \\
\hline & & & & & Pre-dose & $l \mathrm{hr}$ & $24 h r$ & & & & & & & & \\
\hline 1 & $\mathbf{M}$ & 16 & $\begin{array}{l}\text { Day } 1 \\
1 \text { week } \\
2 \text { mth }\end{array}$ & $\begin{array}{l}1.5 \\
1.5 \\
1.5\end{array}$ & $\begin{array}{l}1 / 4 \\
1 / 4 \\
1 / 4\end{array}$ & $\begin{array}{l}1 / 16 \\
1 / 16 \\
1 / 4\end{array}$ & $1 / 4$ & $\begin{array}{r}54 \\
250\end{array}$ & $\begin{array}{r}5.0 \\
16.5\end{array}$ & $\begin{array}{l}3 \cdot 7 \\
3 \cdot 4\end{array}$ & $\begin{array}{l}45 \\
47\end{array}$ & $\begin{array}{l}2 \cdot 0 \\
3 \cdot 1\end{array}$ & $\begin{array}{l}32 \\
25\end{array}$ & $\begin{array}{l}\text { Good } \\
\text { Bad }\end{array}$ & $\begin{array}{l}\text { Unable to work } \\
\text { Drug stopped } \\
\text { one month after } \\
\text { starting therapy, } \\
\text { clinical and } \\
\text { biochemical } \\
\text { deterioration } \\
\text { followed }\end{array}$ \\
\hline 2 & $\mathbf{M}$ & 35 & $\begin{array}{l}\text { Day } 1 \\
1 \text { week } \\
2 \text { mth }\end{array}$ & $\begin{array}{l}1.5 \\
1.5 \\
1.5\end{array}$ & $\begin{array}{l}1 / 2 \\
1 / 4 \\
1 / 4\end{array}$ & $\begin{array}{l}1 / 16 \\
1 / 16 \\
1 / 8\end{array}$ & $1 / 4$ & $\begin{array}{l}15 \\
35\end{array}$ & $\begin{array}{l}1 \cdot 2 \\
2 \cdot 6\end{array}$ & $\begin{array}{l}4 \cdot 0 \\
2 \cdot 9\end{array}$ & $\begin{array}{l}45 \\
48\end{array}$ & $\begin{array}{l}2 \cdot 4 \\
2 \cdot 1\end{array}$ & $\begin{array}{l}18 \\
19\end{array}$ & $\begin{array}{l}\text { Good } \\
\text { Fair }\end{array}$ & $\begin{array}{l}\text { Unable to work } \\
\text { Physical activity } \\
\text { unchanged; } \\
\text { clinical and } \\
\text { biochemical } \\
\text { deterioration }\end{array}$ \\
\hline \multirow[t]{2}{*}{3} & $\mathbf{F}$ & 48 & Day 1 & 1.5 & 1,4 & $1 / 16$ & $1 / 4$ & 67 & $1 \cdot 8$ & $3 \cdot 7$ & 100 & $2 \cdot 3$ & 28 & Good & $\begin{array}{l}\text { Unable to do } \\
\text { housework }\end{array}$ \\
\hline & & & $\begin{array}{l}1 \text { week } \\
1 \text { week }\end{array}$ & $\begin{array}{l}1 \cdot 5 \\
3 \cdot 0\end{array}$ & $\begin{array}{l}1 / 4 \\
1 / 4\end{array}$ & $\begin{array}{l}1 / 16 \\
1 / 64\end{array}$ & & & & & & & & & \\
\hline \multirow[t]{3}{*}{4} & $\mathbf{F}$ & 12 & $\begin{array}{l}2 \text { mth } \\
\text { Day } 1\end{array}$ & - & - & - & $\overline{1 / 4}$ & $\overline{100}$ & - & $\overline{3 \cdot 4}$ & - & $\overline{2 \cdot 6}$ & $\overline{43}$ & Fair & $\begin{array}{l}\text { Lost to follow up } \\
\text { Unable to } \\
\text { attend school }\end{array}$ \\
\hline & & & $\begin{array}{l}1 \text { week } \\
2 \text { mth }\end{array}$ & $\begin{array}{l}1 \cdot 5 \\
1 \cdot 5\end{array}$ & $\begin{array}{l}1 / 4 \\
1 / 2\end{array}$ & $\begin{array}{l}1 / 4 \\
1 / 16\end{array}$ & & 39 & 0.5 & $5 \cdot 0$ & 100 & $1 \cdot 8$ & 36 & Good & $\begin{array}{l}\text { Physical activity } \\
\text { unchanged; } \\
\text { biochemical } \\
\text { improvement }\end{array}$ \\
\hline & & & $5 \mathrm{mth}$ & 3.0 & $1 / 2$ & $1 / 64$ & & 20 & 0.5 & $5 \cdot 5$ & 100 & $1 \cdot 2$ & 28 & Good & No change \\
\hline \multirow[t]{2}{*}{5} & $\mathbf{F}$ & 20 & Day 1 & $1 \cdot 5$ & $1 / 4$ & $1 / 4$ & $1 / 4$ & 250 & 3.0 & $3 \cdot 3$ & 40 & 4.9 & 32 & Fair & $\begin{array}{l}\text { Unable to work; } \\
\text { ascites, oedema }\end{array}$ \\
\hline & & & $\begin{array}{l}1 \text { week } \\
2 \mathrm{mth}\end{array}$ & $\begin{array}{l}1 \cdot 5 \\
1 \cdot 5\end{array}$ & $\begin{array}{l}1 / 4 \\
1 / 2\end{array}$ & $\begin{array}{l}1 / 4 \\
1 / 16\end{array}$ & & 72 & 1.6 & $4 \cdot 2$ & 100 & $4 \cdot 0$ & 30 & Good & $\begin{array}{l}\text { Employed (sales- } \\
\text { girl); clinical and } \\
\text { biochemical } \\
\text { improvement }\end{array}$ \\
\hline \multirow[t]{2}{*}{6} & $\mathbf{M}$ & 39 & $\begin{array}{l}\text { Day } 1 \\
1 \text { week }\end{array}$ & $\begin{array}{l}1 \cdot 5 \\
1 \cdot 5\end{array}$ & $\begin{array}{l}1 / 2 \\
1 / 2\end{array}$ & $\begin{array}{l}1 / 8 \\
1 / 8\end{array}$ & $1 / 2$ & 58 & $2 \cdot 5$ & 3.8 & 38 & $1 \cdot 4$ & 36 & Fair & Unable to work \\
\hline & & & $2 \mathrm{mth}$ & - & - & - & - & - & - & 一 & 一 & - & - & - & Lost to follow up \\
\hline \multirow[t]{2}{*}{7} & $\mathbf{M}$ & 50 & $\begin{array}{l}\text { Day } 1 \\
1 \text { week }\end{array}$ & $1 \cdot 5$ & $1 / 4$ & $1 / 4$ & $1 / 4$ & 250 & 3.5 & $3 \cdot 2$ & & $2 \cdot 0$ & 20 & Fair & $\begin{array}{l}\text { Self employed } \\
\text { (poultry farmer) }\end{array}$ \\
\hline & & & $2 \mathrm{mth}$ & - & - & - & - & - & - & - & - & - & - & - & Lost to follow up \\
\hline \multirow[t]{3}{*}{8} & $\mathbf{F}$ & 21 & Day 1 & $1 \cdot 5$ & $1 / 2$ & $1 / 2$ & $1 / 2$ & 315 & $15 \cdot 5$ & $2 \cdot 1$ & 35 & 3.9 & 18 & Bad & $\begin{array}{l}\text { Limited duties, } \\
\text { ascites }\end{array}$ \\
\hline & & & $\begin{array}{l}1 \text { week } \\
1 \text { week }\end{array}$ & $\begin{array}{l}1 \cdot 5 \\
3 \cdot 0\end{array}$ & $\begin{array}{l}1 / 2 \\
1 / 2\end{array}$ & $\begin{array}{l}1 / 2 \\
1 / 2\end{array}$ & & & & & & & & & \\
\hline & & & $2 \mathrm{mth}$ & - & - & - & - & - & - & - & - & - & - & - & $\begin{array}{l}\text { Drug discon- } \\
\text { tinued, deepening } \\
\text { jaundice }\end{array}$ \\
\hline 9 & $\mathbf{F}$ & 22 & $\begin{array}{l}\text { Day } 1 \\
1 \text { week } \\
2 \text { mth }\end{array}$ & $\begin{array}{l}1 \cdot 5 \\
1 \cdot 5 \\
1 \cdot 5\end{array}$ & $\begin{array}{l}1 / 4 \\
1 / 4 \\
1 / 4\end{array}$ & $\begin{array}{l}1 / 4 \\
1 / 4 \\
1 / 4\end{array}$ & $1 / 4$ & $\begin{array}{r}196 \\
86\end{array}$ & $\begin{array}{r}3 \cdot 6 \\
15 \cdot 3\end{array}$ & $\begin{array}{l}2 \cdot 0 \\
1.9\end{array}$ & $\begin{array}{l}39 \\
24\end{array}$ & $\begin{array}{l}3 \cdot 7 \\
3 \cdot 4\end{array}$ & $\begin{array}{l}43 \\
18\end{array}$ & $\begin{array}{l}\text { Bad } \\
\text { Bad }\end{array}$ & $\begin{array}{l}\text { Unable to work, } \\
\text { ascites } \\
\text { Physical activity } \\
\text { unchanged; } \\
\text { ascites, porto- } \\
\text { systemic encepha- } \\
\text { lopathy deteriora- } \\
\text { tion, died soon } \\
\text { afterwards }\end{array}$ \\
\hline \multirow[t]{3}{*}{10} & $\mathbf{M}$ & 43 & Day 1 & $1 \cdot 5$ & $1 / 4$ & $1 / 4$ & $1 / 4$ & 430 & $10 \cdot 0$ & $2 \cdot 5$ & 100 & $3 \cdot 5$ & 19 & Bad & $\begin{array}{l}\text { Employed } \\
\text { (office work) }\end{array}$ \\
\hline & & & $\begin{array}{l}1 \text { week } \\
2 \mathrm{mth}\end{array}$ & $\begin{array}{l}1.5 \\
1.5\end{array}$ & $\begin{array}{l}1 / 4 \\
1 / 4\end{array}$ & $\begin{array}{l}1 / 4 \\
1 / 4\end{array}$ & & 310 & $3 \cdot 8$ & $2 \cdot 6$ & 100 & $4 \cdot 0$ & 23 & Fair & $\begin{array}{l}\text { Physical activity } \\
\text { unchanged, } \\
\text { biochemical } \\
\text { improvement }\end{array}$ \\
\hline & & & $6 \mathrm{mth}$ & 3.0 & $1 / 4$ & $1 / 4$ & & 208 & 3.0 & $3 \cdot 2$ & 100 & $3 \cdot 6$ & 20 & Fair & $\begin{array}{l}\text { Physical activity } \\
\text { unchanged }\end{array}$ \\
\hline
\end{tabular}

Table II Hepatic function and titre of immunosuppressive activity associated with azathioprine in patients with active chronic hepatitis 


\begin{tabular}{|c|c|c|c|c|c|c|c|c|c|c|c|c|c|c|c|c|}
\hline \multirow[t]{2}{*}{ No. } & \multirow{2}{*}{\multicolumn{3}{|c|}{$\begin{array}{c}\text { Sex Age Time of } \\
\text { (yr) Study }\end{array}$}} & \multirow{2}{*}{$\begin{array}{l}\text { Azathio- } \\
\text { prine } \\
(\mathrm{mg} / \mathrm{kg})\end{array}$} & \multicolumn{3}{|c|}{$\begin{array}{l}\text { Titre of Immunosuppressive } \\
\text { Activity }\end{array}$} & \multirow{2}{*}{$\begin{array}{l}\text { e Bilirubin } \\
(m g / 100 \\
-m l)\end{array}$} & \multirow{2}{*}{$\begin{array}{l}\text { Albumin } \\
(\mathrm{g} / 100 \\
\mathrm{ml})\end{array}$} & \multirow{2}{*}{$\begin{array}{l}\text { Throm- } \\
\text { botest } \\
(\%)\end{array}$} & \multirow{2}{*}{$\begin{array}{l}\gamma-G l o b u- \\
\operatorname{lin}(g / 100 \\
m l)\end{array}$} & \multirow{2}{*}{$\begin{array}{l}\text { Choles- } \\
\text { terol } \\
(\mathrm{mg} / 100 \\
\mathrm{ml})\end{array}$} & \multirow{2}{*}{$\begin{array}{l}\text { Alkaline } \\
\text { Phospha- } \\
\text { tase (KA } \\
\text { units) }\end{array}$} & \multirow{2}{*}{$\begin{array}{l}\text { Antimito- } \\
\text { chondrial } \\
\text { Antibody }\end{array}$} & \multirow[t]{2}{*}{$\begin{array}{l}\text { Liver }^{1} \\
\text { Biopsy }\end{array}$} & \multirow[t]{2}{*}{ Comment } \\
\hline & & & & & Pre-dose & $1 \mathrm{Hr}$ & $24 \mathrm{Hr}$ & & & & & & & & & \\
\hline \multirow[t]{2}{*}{11} & $\mathbf{F}$ & 46 & $\begin{array}{l}\text { Day } 1 \\
1 \text { week }\end{array}$ & $\begin{array}{l}1.5 \\
1.5\end{array}$ & $\begin{array}{l}1 / 2 \\
1 / 2\end{array}$ & $\begin{array}{l}1 / 16 \\
1 / 16\end{array}$ & $1 / 2$ & 0.5 & $3 \cdot 1$ & 64 & $1 \cdot 2$ & 320 & 110 & Positive & Diagnostic & \\
\hline & & & $2 \mathrm{mth}$ & 1.5 & $1 / 2$ & $1 / 16$ & & 0.5 & $3 \cdot 4$ & 100 & 1.7 & 335 & 68 & & & \\
\hline \multirow[t]{2}{*}{12} & $\mathbf{F}$ & 53 & $\begin{array}{l}\text { Day } 1 \\
1 \text { week }\end{array}$ & $\begin{array}{l}1.5 \\
1.5\end{array}$ & $* 2$ & 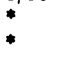 & & 0.5 & $4 \cdot 5$ & 100 & 1.4 & 385 & 14 & Positive & Compatible & \\
\hline & & & $2 \mathrm{mth}$ & 1.5 & $1 / 4$ & $1 / 16$ & & 0.5 & $3 \cdot 7$ & 100 & 1.4 & 410 & 16 & & & \\
\hline \multirow[t]{3}{*}{13} & $\mathbf{F}$ & 64 & Day 1 & 1.5 & $1 / 4$ & $1 / 16$ & $1 / 4$ & 1.8 & $3 \cdot 3$ & 100 & $2 \cdot 1$ & 230 & 80 & Positive & Compatible & $\begin{array}{l}\text { Because } \\
\text { thrombo } \\
\text { openia th } \\
\text { apy was i } \\
\text { mittent }\end{array}$ \\
\hline & & & 1 week & 1.5 & * & * & & & & & & & & & & \\
\hline & $\mathbf{F}$ & & 2 mth & 1.5 & $1 / 4$ & $1 / 16$ & & $1 \cdot 6$ & $3 \cdot 5$ & - & $2 \cdot 8$ & 380 & 65 & & & \\
\hline \multirow{2}{*}{14} & $\mathbf{F}$ & 55 & $\begin{array}{l}\text { Day } 1 \\
1 \text { week }\end{array}$ & $\begin{array}{l}1.5 \\
1.5\end{array}$ & ${ }^{1 / 2}$ & ${ }^{1 / 2}$ & $1 / 2$ & $2 \cdot 8$ & $2 \cdot 2$ & 100 & $2 \cdot 9$ & 190 & 75 & Negative & Diagnostic & \\
\hline & & & $2 \mathrm{mth}$ & 1.5 & $1 / 2$ & $1 / 2$ & & $6 \cdot 0$ & $2 \cdot 3$ & 100 & $2 \cdot 8$ & 200 & 50 & & & \\
\hline \multirow[t]{2}{*}{15} & $\mathbf{F}$ & 35 & Day 1 & 1.5 & $1 / 4$ & $1 / 4$ & $1 / 4$ & $8 \cdot 8$ & 3.8 & 100 & 1.5 & 990 & 72 & Positive & Compatible & $\begin{array}{l}\text { Returned } \\
\text { Greece }\end{array}$ \\
\hline & & & 1 week & $\begin{array}{l}1.5 \\
3.0 \\
-\end{array}$ & $\begin{array}{l}1 / 4 \\
1 / 4 \\
-\end{array}$ & $\begin{array}{l}1 / 4 \\
1 / 4 \\
\end{array}$ & & & & & & & & & & \\
\hline 16 & $\mathbf{F}$ & 65 & $\begin{array}{l}2 \text { mth } \\
\text { Day } 1 \\
1 \text { week }\end{array}$ & $\begin{array}{ll}\overline{1 \cdot 5} & \overline{1} \\
\text { Patient de }\end{array}$ & $\begin{array}{r}\overline{1 / 4} \\
\text { dead }\end{array}$ & $\overline{1 / 4}$ & $\overline{1 / 4}$ & $\overline{11 \cdot 6}$ & $\overline{2 \cdot 4}$ & $\overline{76}$ & $\overline{1 \cdot 2}$ & $\overline{220}$ & $\overline{70}$ & Positive & Diagnostic & $\begin{array}{l}\text { Ascites, } \\
\text { porto- } \\
\text { systemic } \\
\text { encephalo- } \\
\text { pathy }\end{array}$ \\
\hline
\end{tabular}

Table III Hepatic function and titre of immunosuppressive activity associated with azathioprine in patients with primary biliary cirrhosis

'Diagnostic refers to the presence of a florid duct lesion (stage 1) and compatible to the presence of stage 2, 3, or 4 lesions (Scheuer, 1968).

* 2 Blood samples unavailable for testing.

\begin{tabular}{|c|c|c|c|c|c|c|c|c|c|c|c|c|}
\hline \multirow[t]{2}{*}{ No. } & \multirow[t]{2}{*}{ Sex } & \multirow{2}{*}{\multicolumn{2}{|c|}{ Age Diagnosis }} & \multicolumn{3}{|c|}{$\begin{array}{l}\text { Titre of Immuno- } \\
\text { suppressive Activity }\end{array}$} & \multirow[t]{2}{*}{$\begin{array}{l}A s T \\
(i u / l)\end{array}$} & \multirow{2}{*}{$\begin{array}{l}\text { Bilirubin } \\
(m g / \\
100 m l)\end{array}$} & \multirow[t]{2}{*}{$\begin{array}{l}\text { Albumin } \\
(\mathrm{g} / 100 \mathrm{ml})\end{array}$} & \multirow[t]{2}{*}{$\begin{array}{l}\text { Thrombo- } \\
\text { test }(\%)\end{array}$} & \multirow[t]{2}{*}{ Liver Biopsy } & \multirow[t]{2}{*}{ Comment } \\
\hline & & & & $\begin{array}{l}\text { Before } \\
\text { Dose }\end{array}$ & $\begin{array}{l}\text { One } \\
\text { Hr }\end{array}$ & $24 \mathrm{Hr}$ & & & & & & \\
\hline 17 & $\mathbf{F}$ & 20 & Wilson's disease & $1 / 4$ & $1 / 32$ & $1 / 4$ & 9 & $1 \cdot 5$ & $4 \cdot 1$ & 100 & Normal & $\begin{array}{l}\text { Asymptomatic, in } \\
\text { remission, off penicil- } \\
\text { lamine } 10 \text { days }\end{array}$ \\
\hline $\begin{array}{l}18 \\
19\end{array}$ & $\begin{array}{l}\mathbf{F} \\
\mathbf{F}\end{array}$ & $\begin{array}{l}41 \\
48\end{array}$ & $\begin{array}{l}\text { Acute viral } \\
\text { hepatitis } \\
\text { Alcoholic } \\
\text { cirrhosis }\end{array}$ & $\begin{array}{l}1 / 2 \\
1 / 4 \\
1 / 4\end{array}$ & $\begin{array}{l}1 / 4 \\
1 / 32 \\
1 / 8\end{array}$ & $\begin{array}{l}1 / 4 \\
1 / 4 \\
1 / 4\end{array}$ & $\begin{array}{r}115 \\
35 \\
26\end{array}$ & $\begin{array}{l}9 \cdot 0 \\
3 \cdot 8 \\
1 \cdot 6\end{array}$ & $\begin{array}{l}2 \cdot 9 \\
3 \cdot 7 \\
2 \cdot 8\end{array}$ & $\begin{array}{r}49 \\
100 \\
66\end{array}$ & $\begin{array}{l}\text { Acute cholestatic } \\
\text { hepatitis } \\
\text { Micronodular } \\
\text { cirrhosis }\end{array}$ & $\begin{array}{l}\text { This patient was studied } \\
\text { twice, two weeks apart. } \\
\text { Ascites }\end{array}$ \\
\hline 20 & $\mathbf{F}$ & 48 & $\begin{array}{l}\text { Budd-Chiari } \\
\text { syndrome }\end{array}$ & $1 / 4$ & $1 / 4$ & $1 / 4$ & 19 & $4 \cdot 4$ & $2 \cdot 4$ & 65 & $\begin{array}{l}\text { Acute Budd- } \\
\text { Chiari }\end{array}$ & $\begin{array}{l}\text { Ascites, died soon } \\
\text { afterwards }\end{array}$ \\
\hline
\end{tabular}

Table IV Hepatic function and titre of immunosuppressive activity associated with azathioprine in patients with miscellaneous liver disease

hepatic function (nos. 4 and 5); the other patient (no. 10) had improved from bad to fair. Again the titre of serum immunosuppressive activity was related to hepatic function. Patient no. 1, who had initially possessed good hepatic function and was then able to activate the drug normally, now had bad hepatic function and failed to raise his titre above pretreatment values. Patient no. 2 had also deteriorated but to a lesser degree. Upon retesting his titre was lower than when initially tested and less than normal. Patients nos. 4 and 5 had improved drama- tically. On initial testing neither was able to activate the drug but on retesting they had achieved normal titres. Patients nos. 9 and 10 on retesting after two months of therapy again failed to elevate their titres above pretest levels. One had deteriorated (no. 9) and although the other patient (no. 10) had improved, his hepatic function was still only fair. Thus in summary: (1) Two patients who failed to respond to therapy did not develop immunosuppressive activity either initially or when retested after two months of therapy. (2) Two patients who initially failed to 
develop immunosuppressive activity nevertheless responded to therapy and on retesting developed normal immunosuppressive activity. (3) Two patients who initially developed normal immunosuppressive activity failed to respond to therapy (in one the period of therapy was not continuous and was about one month in duration). On retesting neither achieved normal levels of serum immunosuppressive activity.

\section{Patients with primary biliary cirrhosis}

After two months of continuous medication there was remarkably little change in hepatic function in the four patients available for retesting. One patient (no. 14) showed a steep rise in her serum bilirubin from $2 \cdot 8$ to $6.0 \mathrm{mg} / 100 \mathrm{ml}$ but her other indices of hepatic function were not much different from the values recorded on the commencement of therapy. Assessment of serum immunosuppressive activity at this time revealed no changes from that noted on initial testing in any patient. Patient no. 12, whose initial samples were not tested, still had good hepatic function and was able to develop immunosuppressive activity normally.

\section{SUBJECTS GIVEN AZATHIOPRINE 3.0 MG/KG BODY WEIGHT}

All the normal subjects given $3.0 \mathrm{mg} / \mathrm{kg}$ of azathioprine achieved much higher titres within one hour of administration of the drug (Table I) than those observed in the same subjects after $1.5 \mathrm{mg} / \mathrm{kg}$. This indicated that the larger dose produced greater levels of circulating active drug in the serum. Of the patients tested in a similar manner, two (nos. 3 and 4) had good hepatic function at the time and achieved normal titres of immunosuppressive activity (Table II). The other three patients had fair or bad hepatic function and failed to elevate their titres above that attained after $1.5 \mathrm{mg} / \mathrm{kg}$ of azathioprine.

\section{Discussion}

These results confirm those of Bach and his coworkers (Bach and Dardenne, 1970) and Mitchell and her associates (Mitchell, Eddleston, Smith, and Williams, 1970) that in patients with severe liver disease the metabolism of azathioprine is altered, resulting in a decreased level of circulating biologically active drug as reflected by low titres of immunosuppressive activity in the rosette inhibition test. The titre attained was related to the degree of impairment of hepatic function for patients with mild degrees of hepatic dysfunction developed normal titres, whereas in those with severe liver disease the titre of immunosuppressive activity never rose above pretreatment levels (Tables II, III, and IV). The importance of adequate hepatic function for the development of immunosuppressive activity was also seen in the patients who were studied over a prolonged period of time. As hepatic function improved or deteriorated in these patients, the titres of immunosuppressive activity in the serum rose or fell concomitantly. From the studies with azathioprine $3.0 \mathrm{mg} / \mathrm{kg}$ it can be seen that the titre of immunosuppressive activity attained in patients with adequate function was dose dependent (Table II). However, if hepatic impairment was severe no rise in titre was achieved even with the higher dose of drug (Tables II and III). The development of immunosuppressive activity was not related to the aetiology of the liver disease nor was it influenced by the presence or absence of serum antibodies to smooth muscle, against nuclei or mitochondria.

The mechanism of rosette inhibition activity is complex and as yet incompletely understood, thus there may be many explanations for the observed failure to develop normal immunosuppressive activity after azathioprine in patients with significant impairment of hepatic function. It seems likely that this effect could be due to alterations in the normal pattern of azathioprine metabolism by changes in absorption, activation, or degradation of the drug singly or in combination. Bach and Dardenne (1970) using S-35 labelled and intravenous azathioprine demonstrated that the abnormalities of azathioprine metabolism in patients with liver disease cannot be explained by malabsorption. Likewise, since patients with impaired hepatic function excrete in urine similar quantities of the degradative metabolites of azathioprine (mainly inorganic sulphate, thiouric acid, 6 methyl sulphinyl 8 hydroxy purine) to normal man (Elion, 1972), it can reasonably be assumed that unaltered azathioprine does not continue to circulate in the presence of liver disease. The precise reason for the low immunosuppressive titres in liver disease remains unknown. However, this observation could well result from a change in the ratio of the activation to the degradation of the drug, possibly in the liver.

Only a small number of patients were available for restudy after several months of therapy. None of the patients with primary biliary cirrhosis had experienced any significant alteration in their liver function and therefore it was not surprising that their titres of activity had not changed. However the results in the group with active chronic hepatitis allow certain points to be made. Failure to develop immunosuppressive activity in two patients (nos. 9 and 10) was associated with lack of complete suppression of their disease processes and this might have been predicted. However, a good response in two patients (nos. 4 and 5), despite failure of their titres to rise and the lack of response in one patient (no. 2), even though he developed a normal titre when initially 
tested, was unexpected. As the number of patients observed over a prolonged period was small and as no untreated patients were observed simultaneously, the possibility remains that these observations may have been fortuitous. Likewise, failure to develop immunosuppressive activity in the serum after azathioprine in patients with liver disease does not necessarily mean that activation of the drug does not occur. The clinical and biochemical improvement in patients taking the drug suggests that activation does indeed occur. The results in some of our patients (nos. 4, 5, and possibly 10) are consistent with the possibility that active metabolites of azathioprine were produced in amounts too small to be detected in the serum, but still in sufficient quantities to attain adequate tissue levels after several months of therapy.

These results in patients after two months of therapy suggest that the initial titre of immunosuppressive activity is of no predictive value in estimating the type of response to treatment. Therefore, possibly some action of antimetabolites, unrelated to immunosuppression, may be responsible for the favourable response to therapy in some patients. Perhaps a non-specific anti-inflammatory effect of these agents is important in suppressing the process involved in disorders such as active chronic hepatitis.

The authors wish to thank Dr A. H. Griffith of Wellcome Research Laboratories, Beckenham, Kent, for his assistance in setting up the rosette inhibition test and for English translations of the relevant French literature; Dr J. F. Bach of the Necker Hospital, Paris, for his interest and helpful discussions; Dr R. A. Fox, Dr D. Owens, and Dr A. Ross of the Royal Free Hospital, for their assistance in the follow-up care of the patients.
References

Bach, J. F. (1970). Mechanism and significance of rosette inhibition by antilymphocyte serum. In International Symposium on Antilymphocytic Serum, Versailles, 1970, edited by J. F. Bach et al (Symposia series in Immunoglobulin Standardization, vol. 16), pp. 189-198. Karger, Basle and New York.

Bach, J. F., and Dardenne, M. (1970). Etudes sur le métabolisme de l'azathioprine. C.R. Acad. Sci. Paris D, 271, 453-456.

Bach, J. F., Dardenne, M., and Fournier, C. (1969a). In vitro evaluation of immunosuppressive drugs. Nature (Lond.), 222, 998-999.

Bach, J. F., and Dormont, J. (1971). Further developments of the rosette inhibition test for the testing of antihuman lymphocyte serum. Transplantation, 11, 96-100.

Bach, J. F., Dormont, J., Dandenne, M., and Balner, H. (1969b). In vitro rosette inhibition by antihuman antilymphocyte serum. Transplantation, 8, 265-280.

Elion, G. B. (1967a). Biochemistry and pharmacology of purine analogues. Fed. Proc., 26, 898-903.

Elion, G. B. (1967b). In Immunopathology: Mechanisms of Inflammation Induced by Immune Reactions: 5th International Symposium on Immunopathology, Punta Ala, edited by P. A. Miescher and P. Grabar, pp. 399-401. Grune and Stratton, New York.

Elion, G. B. (1969). Action of purine analogs: enzyme specificity studies as a basis for interpretation and design. Cancer Res., 29, 2448-2453.

Elion, G. B. (1972). Significance of azathioprine metabolites. Proc. roy. Soc. Med., 65, 257-260.

Mackay, I. R. (1968). Chronic hepatitis: effect of prolonged suppressive treatment and comparison of azathioprine with prednisolone. Quart. J. Med., 37, 379-392.

Mackay, I. R., Weiden, S., and Ungar, B. (1964). Treatment of active chronic hepatitis and lupoid hepatitis with 6-mercaptopurine and azathioprine. Lancet, 1, 899-902.

Mistilis, S. P., and Blackburn, C. R. B. (1967). The treatment of active chronic hepatitis with 6-mercaptopurine and azathioprine. Aust. Ann. Med., 16, 305-311.

Mitchell, C. G., Eddleston, A. L. W. F., Smith, M. G. M., and Williams, R. (1970). Serum immunosuppressive activity due to azathioprine and its relation to hepatic function after liver transplantation. Lancet, 1, 1196-1199.

Page, A. R., Good, R. A., and Pollara, B. (1969). Long term results of therapy in patients with chronic liver disease associated with hypergammaglobulinemia. Amer. J. Med., 47, 765-774.

Scheuer, P. J. (1968). Liver Biopsy Interpretation. Bailliere, Tindall, Cassell, London.

Siegel, S. (1956). NonParametric Statistics for the Behavioral Sciences. McGraw-Hill, New York. 